\title{
Real-Time Distributed Fiber Optic Sensor for Security Systems: Performance, Event Classification and Nuisance Mitigation
}

\begin{abstract}
Seedahmed S. MAHMOUD, Yuvaraja VISAGATHILAGAR, and Jim KATSIFOLIS*
Future Fibre Technologies Pty Ltd. 10 Hartnett Close, Mulgrave, VIC 3170, Australia

*Corresponding author: Jim KATSIFOLISＥ-mail: jimk@fftsecurity.com

Abstract: The success of any perimeter intrusion detection system depends on three important performance parameters: the probability of detection (POD), the nuisance alarm rate (NAR), and the false alarm rate (FAR). The most fundamental parameter, POD, is normally related to a number of factors such as the event of interest, the sensitivity of the sensor, the installation quality of the system, and the reliability of the sensing equipment. The suppression of nuisance alarms without degrading sensitivity in fiber optic intrusion detection systems is key to maintaining acceptable performance. Signal processing algorithms that maintain the POD and eliminate nuisance alarms are crucial for achieving this. In this paper, a robust event classification system using supervised neural networks together with a level crossings (LCs) based feature extraction algorithm is presented for the detection and recognition of intrusion and non-intrusion events in a fence-based fiber-optic intrusion detection system. A level crossings algorithm is also used with a dynamic threshold to suppress torrential rain-induced nuisance alarms in a fence system. Results show that rain-induced nuisance alarms can be suppressed for rainfall rates in excess of $100 \mathrm{~mm} / \mathrm{hr}$ with the simultaneous detection of intrusion events. The use of a level crossing based detection and novel classification algorithm is also presented for a buried pipeline fiber optic intrusion detection system for the suppression of nuisance events and discrimination of intrusion events. The sensor employed for both types of systems is a distributed bidirectional fiber-optic Mach-Zehnder (MZ) interferometer.
\end{abstract}

Keywords: Adaptive level crossings, fiber optic sensor, intrusion detection, nuisance alarm

\section{Introduction}

Distributed fiber-optic sensors have been used in many commercial and defense applications. These sensors have been used to protect assets such as airports, commercial and defense infrastructure, and oil and pipeline systems. A number of underlying sensing technologies can be implemented when designing distributed fiber-optic sensors which include Mach-Zehnder (MZ) interferometers [1, 2], Michelson interferometers [1], Fiber-Bragg grating arrays [1], Sagnac loops [1], and coherent optical time domain reflectometry (C-OTDR) [3, 4].

High performance distributed fiber-optic sensors have been applied to both outdoor and buried intrusion detection systems in recent years. The advantages of using fiber optic sensors in intrusion detection systems over conventional technologies are well recognized and include their immunity to electromagnetic interference, high sensitivity, no power required in the field, intrinsic safety in volatile environments, and high reliability and cost effectiveness over large distances. Their implementation in noisy or hostile environments

Received: 8 June 2012 / Revised version: 15 June 2012

(C) The Author(s) 2012. This article is published with open access at Springerlink.com 
presents some interesting challenges which need to be overcome in order to achieve acceptable performance. In all outdoor perimeter intrusion detection systems, there exists a performance trade-off between the probability of detection and nuisance alarm rate [5]. These systems are susceptible to a wide range of nuisance alarms from both environmental and man-made sources which can include wind, torrential rain, storms, and nearby traffic crossings.

A number of sensor related signal processing algorithms have been presented in the literature for suppressing nuisance alarms. Jiang et al. [6] proposed a classification method for an MZ interferometric sensor using a wavelet packet transform for denoising and feature extraction and a neural network as a classifier. This method however is not suitable for eliminating nuisance alarms due to torrential rain as the signal amplitude of the sensor would be saturated in the time domain. Vries [7] proposed an acoustic based perimeter intrusion classification system that deployed a neural network with frequency domain features to detect different types of intrusion events such as climbing, cutting and jumping over the fence. The system however suffered from performance degradation when the quality of the sound (SNR) generated by the intruders and the surrounding environment decreased. Moreover, the frequency domain features were not robust enough to distinguish between nuisance and intrusion events.

Yousefi et al. [8] presented a fence breach detection system which could detect activity on the fence and discriminate different types of activity. The hardware of the system comprised a 3 -axis accelerometer and a RISC microprocessor. The system employed an algorithm that detects activity and non-activity on the fence. It also recognized the type of breach whether it was due to rattling caused by strong wind or a person climbing on the fence. This system used signal variation features along with the energy of two bandpass filters to separate the rattle and climb frequency components. While this showed some success, it was not possible to discriminate between classes that had a similar impact on a fence. Moreover, this algorithm was used to classify a small number of classes (limited to two classes). Min et al. [9] proposed a real-time monitoring system using an audio sensor to detect abnormal activity in the vicinity of buried gas pipes. They extracted a frequency domain feature using a nonlinear scale filter bank method and cepstral mean subtraction along with a combination of two classifiers using the Gaussian mixture model and multi-layer perceptron. Their system achieved a $92 \%$ detection rate to abnormal activity such as hammer drilling and digging. The detection rate of intrusion was however degraded in the presence of background noise such as traffic in the vicinity of the sensor.

In this paper, robust level crossings based signal processing algorithms are presented for detecting intrusion event and suppressing nuisance alarms in both outdoor fence-mounted and buried fiber-optic intrusion detection systems without significantly affecting sensitivity. The use of a real-time level crossing algorithm to suppress rain-induced nuisance alarms and discriminate between continuous nuisance and non-continuous intrusion events in perimeter intrusion detection systems is described. The use of a level crossing based detection method and novel classification algorithm is also presented for the suppression and discrimination of nuisance events from intrusion events in a buried intrusion detection system. Results are shown from real-time fiber optic sensing systems.

\section{Fiber optic intrusion detection system}

The intrusion detection system used in this work is based on the future fiber technologies microstrain locator technology as applied to fence perimeter applications [2]. The microstrain locator is based on the use of a bidirectional $\mathrm{MZ}$ as a distributed sensor 
to detect and locate an intrusion anywhere along the sensing length, $L_{S}$, as shown in Fig. 1. The two sensing fibers and the lead out fiber are typically housed in a standard single mode fiber optic cable which is mounted on the perimeter fence. In this paper, the intrusion detection system will be referred to as the locator.

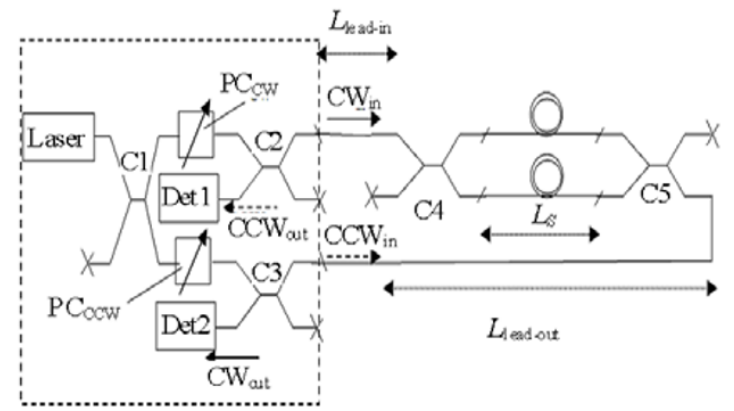

Fig. 1 A basic future fiber technologies microstrain locator system using a bi-directional $\mathrm{MZ}$ with the input polarization control: $\mathrm{C} 1-\mathrm{C} 5$ are all 50:50 fiber couplers.

The deployed sensing system consists of an industrial computer which houses a highly coherent 1550-nm laser source which injects continuous wave counter-propagating light into the MZ. Two detectors, also housed in the sensing controller, receive the clockwise $(\mathrm{CW})$ and counter-clockwise $(\mathrm{CCW})$ signals from the MZ to analyze the signals. The sensing controller also includes polarization controllers $\mathrm{PC}_{\mathrm{CW}}$ and $\mathrm{PC}_{\mathrm{CCW}}$ to maximize the fringe visibility of the $\mathrm{MZ}$ and optimize the location accuracy by actively compensating for changes in fiber birefringence. Detection of an event is based on analyzing the interferometric signals, while the location of an event along the sensing length $L_{S}$ is resolved by measuring the time difference between received counter propagating signals. Additionally, using the event signals detected by both detectors it is possible to apply the appropriate signal processing techniques to classify the signals and perform both event recognition and event discrimination.

\subsection{System installation}

For fence-mounted perimeter systems, the implementation of the sensor as a fence-based perimeter system is achieved by housing the two sensing fibers as well as the insensitive lead-out fiber $L_{\text {lead-out }}$ in a single fiber cable which is directly attached to the fence fabric as shown in Figs. 2 and 3 [10]. This can be applied to a range of fence fabrics including chain link, weld mesh, and palisade styles.

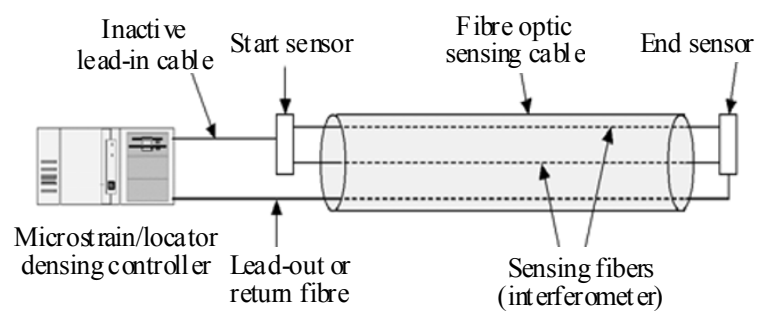

Fig. 2 Use of a single fiber cable to implement the bidirectional MZ sensing system.

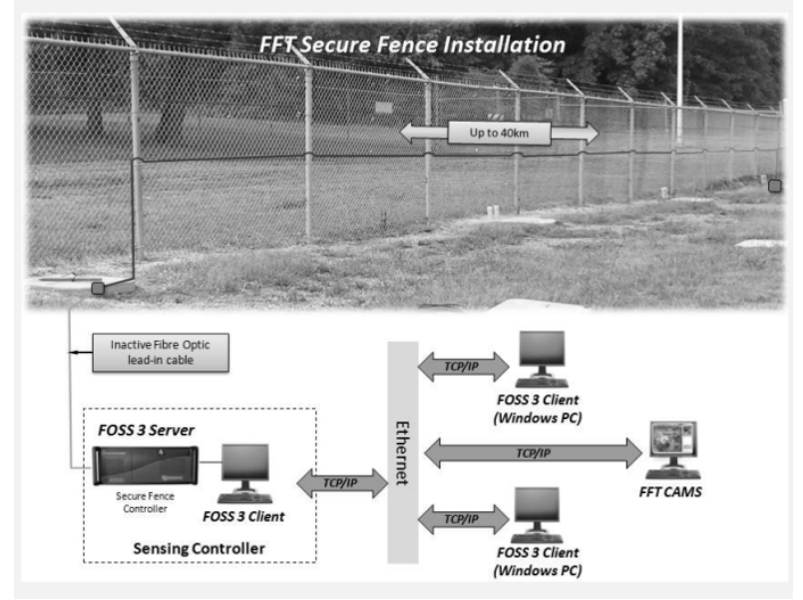

Fig. 3 Implementation of the locator sensor on a fence perimeter.

The quality of installation of the sensing cable on the fence structure is very important in achieving optimum system performance and is very often underestimated. The fence construction needs to be built according to an acceptable standard and the sensing cable attached correctly. Poor fence construction and sensor cable attachment contribute to excessive nuisance alarms and long-term performance degradation. While good installation practices do not eliminate all nuisance alarms, it will ensure that excessive nuisance signals are not generated due to hypersensitivity of the fence to environmental noise. It also enables optimal 
performance of any nuisance mitigation algorithms employed. Another factor which impacts the probability of detection is the cable configuration which is used. Figures 4(a), 4(b), and 4(c) show three different examples of possible installation configurations of the sensor cable on a chain link fence. While the configuration in Fig. 4(a) can provide a basic level of security, looping the sensor up and down the posts [Fig.4(b)] will improve the detection rate of fence climbing at or near the more rigid posts and is a typical configuration used. Where higher sensitivity is required, other cable configurations may be implemented such as an extra loop in the middle of the fence panels [Fig. 4(c)]. The exact configuration used will vary and will depend on the level of security required, the types of intrusion events to be detected, and the skill level of the intruder. It is important that the type of the fence barrier and cable configuration is chosen to match the security requirements of the proposed system.

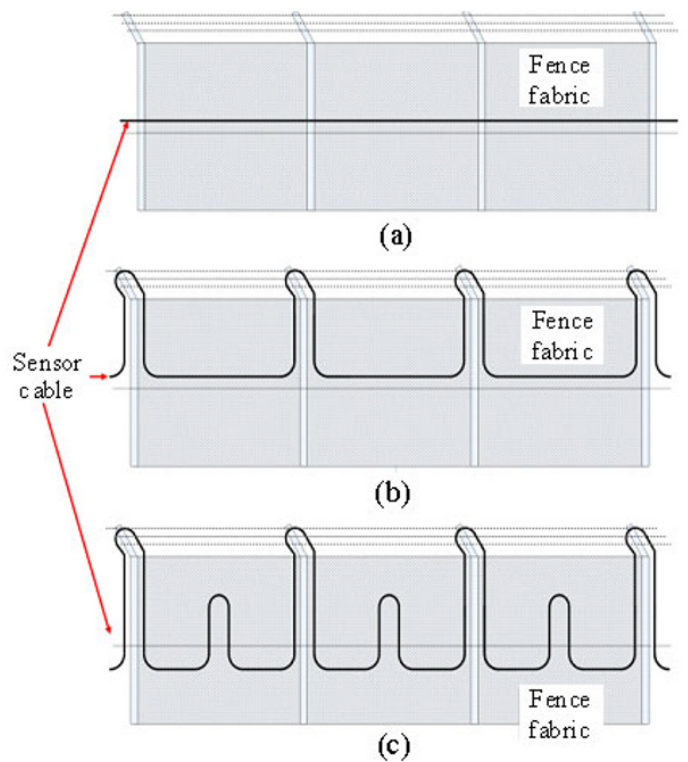

Fig. 4 Example of different cable configurations on a chain link fence.

In buried systems, the sensor cable is configured identical to the fence sensor except the sensing cable is typically buried next to an oil or gas pipeline to detect third party interference (TPI) activities as shown in Fig. 5. Inevitably, it will also be sensitive to other non-intrusion events such as those from nearby traffic and railway crossings.

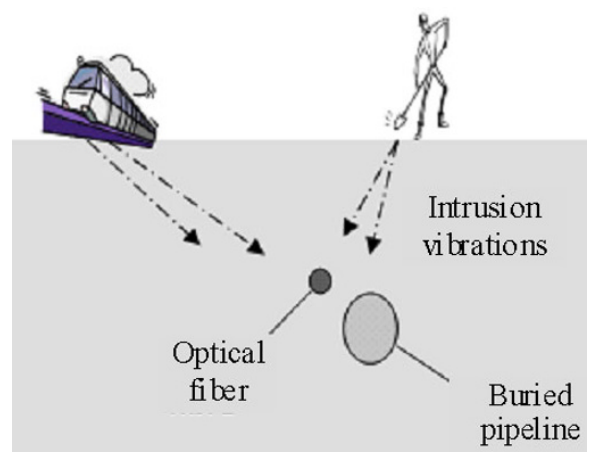

Fig. 5 Cross section of a buried fiber optic intrusion detection system for detection of third party interference.

\subsection{Performance parameters}

The success of any perimeter intrusion detection system depends on three important performance parameters: the probability of detection (POD), the nuisance alarm rate (NAR), and the false alarm rate (FAR). The POD is related to the sensitivity of the system and provides an indication of a system's ability to detect an intrusion within the protected area. A nuisance alarm is any alarm which is generated by an event that is not of interest. A false alarm refers to an alarm generated by the system electronics and is not related to the sensor or an event. False alarms can be minimized through the appropriate system design. Nuisance alarms are typically generated by environmental conditions such as rain, wind, snow, wildlife, and vegetation, as well as man-made sources such as traffic crossings, industrial noises, and other ambient noise sources. While increasing the sensitivity of a system increases its POD, it also increases its sensitivity to nuisance events. Basic event detection algorithms with little event discrimination capability which are applied to a wide range of intrusion events can lead to increased nuisance alarm rates as well as decreased POD. Advanced signal processing algorithms that can maintain a high POD and eliminate nuisance alarms are therefore crucial in perimeter intrusion detection systems. In the following sections, the use of event recognition and 
classification techniques to maintain a high POD and minimize alarms caused by nuisance events will be presented for each type of the system.

\section{Intrusion detection and nuisance suppression in fence systems}

Event classification in perimeter intrusion detection systems can be defined as the categorization of detected signals into identifiable pattern classes through the extraction and analysis of unique signal features and attributes. The selection of unique features from the detected signals is paramount as it ultimately leads to a simplification of the classification problem as well as faster processing and higher detection rates [11]. The main benefits of an accurate and robust event classification system include the ability to discriminate between nuisance events and intrusion events, and more importantly, to suppress nuisance alarms without significantly compromising the probability of detection.

A typical event classification system consists of a pre-processing stage that extracts unique features from the detected event, and a classifier that assigns the computed features to a particular class of intrusion or nuisance (see Fig. 6). Feature samples of nuisance and intrusion events are used to train a classifier offline, and when training is complete, the system will classify new instances based on what is learnt in the training phase. Accurate nuisance and intrusion event classification requires both features that are highly discriminative with respect to the classes of interest and a classifier which can form arbitrary boundaries in the feature space.

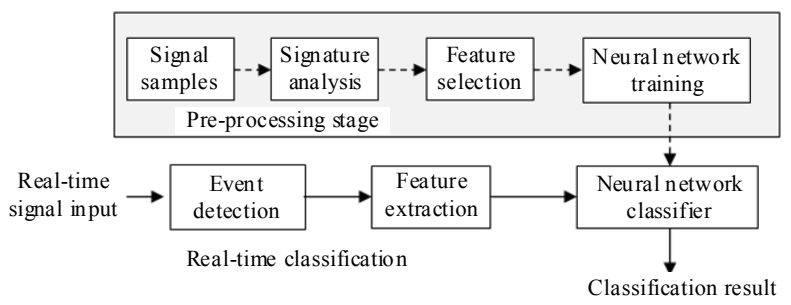

Fig. 6 An event classification system consisting of a pre-processing stage and a real-time classification stage.

\subsection{Neural networks based classification}

Accurate event detection and the use of suitably discriminative features are critical in any event classification application. When training a classifier, the classifier creates boundaries in the features space between the investigated classes. Inaccurate detection of an event can be a common cause of errors in automatic classification [12].

A real-time event detection and feature extraction based on a level crossings (LC) algorithm was proposed and implemented [10]. This algorithm has been used to form the basis of detecting and classifying both intrusion and non-intrusion (nuisance) events. This allows for an effective way of reducing the nuisance alarm rate without reducing the sensitivity of the system. In this work, the intrusion events of interest for fence perimeter systems are fence-climbing and fence-cutting, while the nuisance events which need to be recognized and discriminated are throwing a stone at the fence (stone-throwing) and dragging a stick along the fence (stick-dragging).

In general, the LC algorithm can be defined by the number of crossings, in the positive direction, of an input vector through a given threshold and can be expressed as $[10,13]$

$$
L C=\sum_{n=0}^{N-1} \Psi\{(x(n) \geqslant \text { thresh }) \&(x(n-1)<\text { thresh })\}
$$

where $x$ is a signal of the length $N$, the parameter "thresh" is the level threshold, and the indicator function $\Psi$ is 1 if its argument is true, or 0 otherwise. The level threshold is always positive and just above the system noise.

Based on the level crossings representation of the intrusion signal, a number of configurable parameters can be defined to detect events in real time [10]:

1. Trigger level (TL): the level at which the intrusion event will be detected.

2. Zero settle blocks (ZSB): the number of blocks with zero values before and after an event to determine the start and end of the event. 
3. Zero level (ZL): the level crossing per block below which will be considered as zero.

4. Maximum duration (MD) of the event: limits the duration of a continuous event such as stick dragging on the fence.

Figures 7(a), 7(b), and 8 show examples of a climb event, cut event and stick drag event detected by the proposed detection algorithm, respectively. The TL parameter was set to $5 \mathrm{LCs}$, the MD was set to 46 blocks, and the ZSB was set to 5 blocks.

Figure 8 shows the termination of the long event after it satisfies the MD parameter. Features are extracted from these detected events and fed into neural networks.
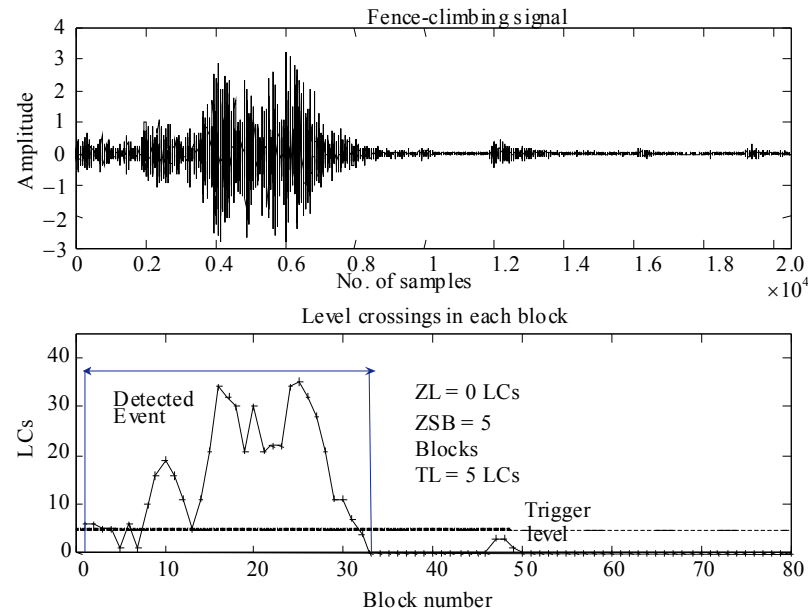

(a)

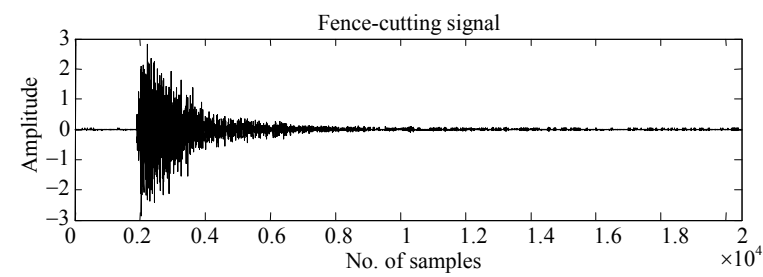

Level crossings in each block

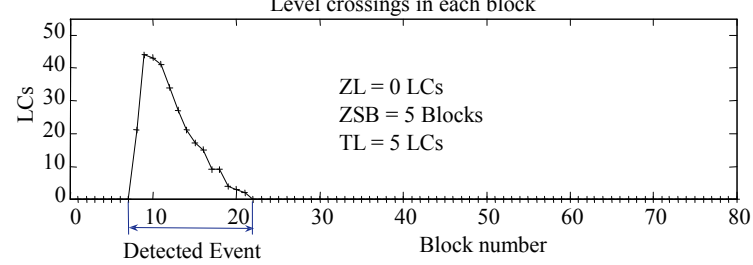

(b)

Fig. 7 Time domain and LC representation of (a) a climb event and (b) cut event detected by the proposed algorithm.
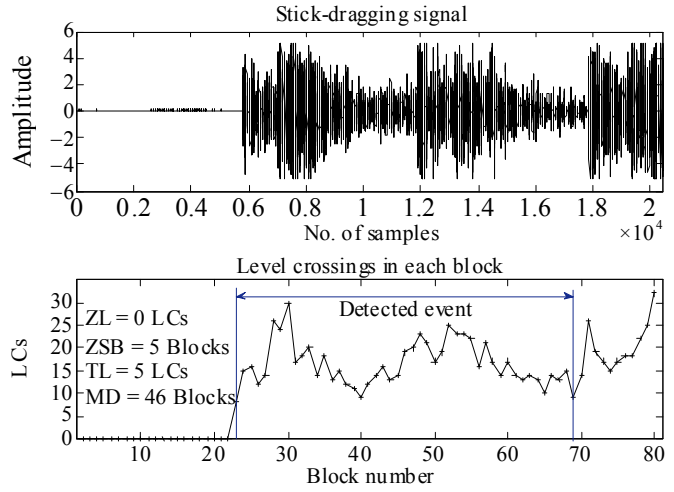

Fig. 8 Time domain and LC representation of a stick drag event detected by the proposed algorithm.

A time-domain signal contains too much irrelevant data to be used directly for classification [11]. Additional features are usually required for an effective classification. Using the LC algorithm, five features have been extracted from the LC representation of the detected intrusion signals. These features are:

1. Total level crossings (TLC): the total number of level crossings for the duration of the event.

2. Duration $(d)$ : the duration of the detected event as a number of blocks (see Fig. 9).

3. Slope of the falling edge of the LCs: the slope of the falling edge of the LC graph as measured between the line formed when joining the end point of the duration $\left(x_{1}, y_{1}\right)$ and the point $\left(x_{2}, y_{2}\right)$. In Fig. 9 which represents a climbing event, a threshold has been used to select the point $\left(x_{2}, y_{2}\right)$.

4. The angle (slope) of the rising edge of the LCs, $\theta$ : the angle of the rising slope edge, $\theta$, is measured between the line formed when joining the initial point of the duration and the maximum point on the LCs graph, and the $x$-axis (see Fig. 9). In most of the cutting events, the rising edge of the LCs forms approximately a right angle $\left(90^{\circ}\right)$ with the $x$-axis, while the climbing events form an acute angle $\left(<90^{\circ}\right)$. This feature is very important for the climbing event and cut event classification.

5. Number of zeros (NZ): the number of zeros (NZ) is the number of blocks within the detected events which have a value less than the ZL parameter. 

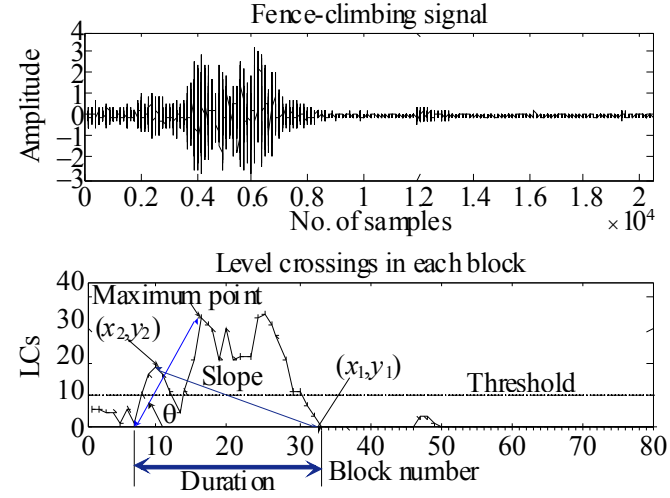

Fig. 9 Representation of the extracted features from the LCs graph of a climbing event.

A three-layer supervised artificial neural network (ANN) with a back-propagation learning algorithm was used to classify the detected events. The five extracted LC based features were used as inputs to the ANN. The advantage of using an ANN is that it can be trained and can implement fast decision algorithms making them suitable for real-time applications [14, 15]. Additionally, event classification by the ANN does not require any statistical assumptions regarding the data and can learn to recognize the characteristic features of the data to classify the event efficiently and accurately.

The LCs and neural network based classification system described was implemented in a chain link fence-mounted fiber-optic intrusion detection system based on the locator technology with a sensing length of $600 \mathrm{~m}$. The extracted LC based features were normalized and used as inputs to the feed-forward neural networks with an input layer consisting of 5 neurons, a hidden layer with 3 neurons, and an output layer consisting of 2 neurons. Performance of the classification system was measured against fence-climb, fence-cut, stick dragging, and stone-throwing classes. The ANN was trained with training data representing these classes, and the weights were stored for real-time testing. Fifteen training events were used per class.

The total number of tested events for each class was 26 . Table 1 shows the impact of the proposed features on the performance of the classification system. From the table, it can be observed that while using four of the extracted LC features produces an optimum result for fence cutting and stone throwing events, by using all five features, it is possible to achieve a $100 \%$ correct classification rate for all four events. The classification accuracy of the system is given by

$$
\text { classification accuracy }=\frac{t}{n} * 100 \%
$$

where $t$ is the number of events correctly classified, and $n$ is the total number of tested events per class.

Table 1 Impact of the proposed features on the performance of the classification system.

\begin{tabular}{c|c|c|c|c}
\hline \multicolumn{5}{c}{ Performance in } \\
\hline Features & Climb & Cut & Stick drag & Stone throwing \\
\hline TLC, $d$, slope, $\theta$ & $87 \%$ & $100 \%$ & $95 \%$ & $100 \%$ \\
\hline TLC, $d$, slope, $\theta$, NZ & $100 \%$ & $100 \%$ & $100 \%$ & $100 \%$ \\
\hline
\end{tabular}

\subsection{Mitigation of continuous-nuisance alarm based on LCs}

Suppression of continuous nuisance alarms such as those induced by torrential rain is one of the most challenging tasks for outdoor perimeter intrusion detection systems (PIDS). A real-time level crossings algorithm to mitigate rain-induced nuisance alarms in fence based fiber-optic intrusion detection systems was proposed and implemented [13]. This algorithm is computationally non-intensive, and it can be used to eliminate rain-induced nuisance alarms for torrential rainfall rates up to and in excess of $100 \mathrm{~mm} / \mathrm{hr}$. The LCs based algorithm is also used to discriminate between continuous nuisances such as rain and non-continuous intrusion events, which allows for simultaneous detection of intrusion events. The algorithm also employs a dynamic event threshold to be able to automatically adjust to varying rainfall rates.

The LC algorithm was integrated into the locator sensing controller which was installed in numerous sites worldwide that experienced torrential rainfall. 
These sites experienced rainfall rates up to and in excess of $100 \mathrm{~mm} /$ hour ( $>4$ inches $/ \mathrm{hr}$ ). Results from these sites have demonstrated the elimination of rain-induced nuisance alarms with the simultaneous detection of intrusion events. The LC-based algorithm is used to discriminate between continuous nuisances such as rain and non-continuous intrusion events. Due to its continuous nature, torrential rainfall will generate a fairly consistent level-crossing rate per time block period. This feature can be used to suppress rainfall induced alarms from the system. By monitoring for any changes in the level crossing rate, non-continuous intrusion events such as fence climbing or cutting can be detected during the rainfall period. Using a dynamic intrusion event threshold has also proven to be effective in automatically adjusting to variable rainfall rates. Figure 10 shows an example of the detected torrential rain signal on a $3.2-\mathrm{km}$ long chain link fence perimeter. The LC representation (see inset nuisance level graph in Fig. 10) can be used to detect intrusions whose signals are essentially buried inside the time domain representation of the rain signal. The LC algorithm can also be adapted to deal with other continuous or semi-continuous nuisances such as nearby traffic noise in a similar way.

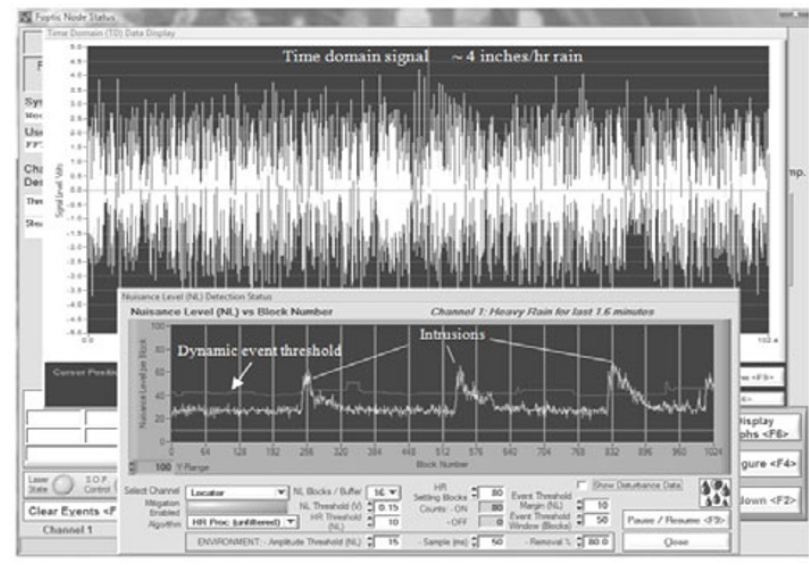

Fig. 10 Real-time elimination of rain-induced nuisance alarms with simultaneous intrusion detection on a $3.2-\mathrm{km}$ chain link fence (the inset graph is the LC representation of nuisance and intrusion events).

\section{Intrusion detection and nuisance suppression in buried systems}

Buried-fiber-optic sensors, such as those implemented for protecting buried oil and gas pipelines, are designed to detect physical disturbances generated by TPI which includes accidental or deliberate digging or excavation activities. These systems are also susceptible to a range of ground based nuisance events such as road and railway traffic and other nearby construction activities. These events can reduce an intrusion detection system's effectiveness with an unacceptably high rate of nuisance alarms. In this section, some novel signal processing techniques are outlined to mitigate the effect of these nuisance events on buried intrusion detection systems by suppressing particular nuisance induced alarms without affecting alarms generated by intrusion events of interest. Figures 11(a) and 11(b) show detected signal examples of typical intrusion events that should be detected, while Figs. 12(a) and 12(b) show typical signals of nuisance alarms due to traffic that should be rejected on a $2.7-\mathrm{km}$ buried gas pipeline.

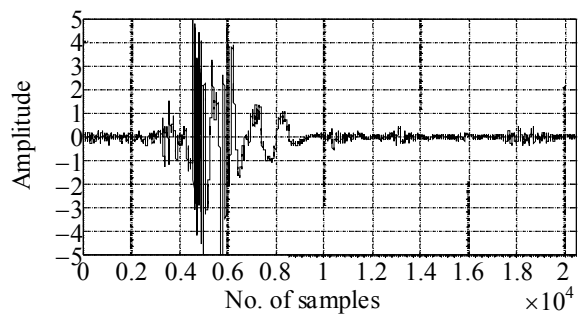

(a)

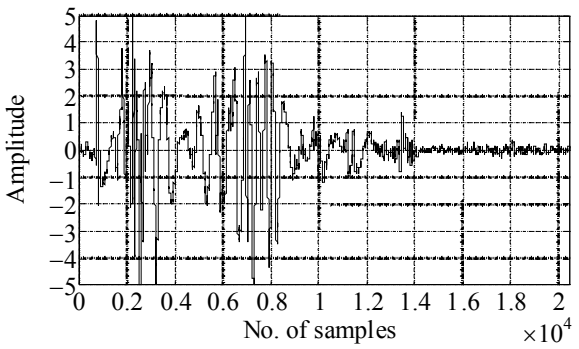

(b)

Fig. 11 Time domain representation of intrusion events: (a) the intrusion signal caused by digging with a pick-axe above a $2.7-\mathrm{km}$ buried gas pipeline protected by a locator system and (b) intrusion signal caused by digging with a back-hoe above a 2.7-km buried gas pipeline protected by a locator system. 


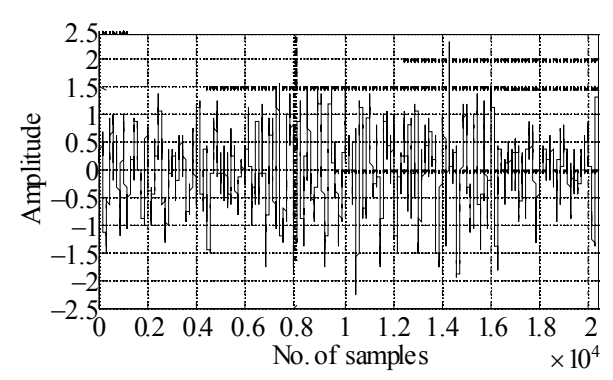

(a)

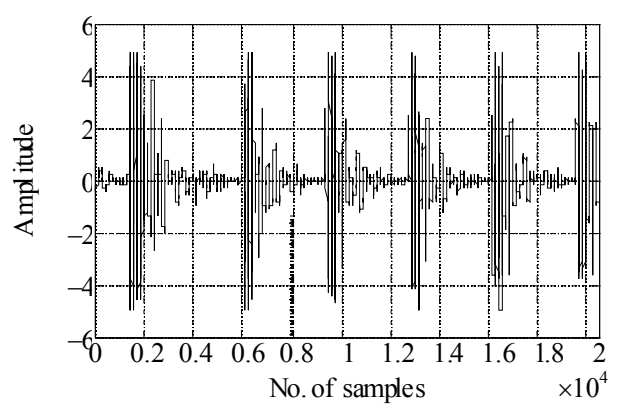

(b)

Fig. 12 Time domain representation of nuisance events: (a) the nuisance signal from traffic on a nearby road for a 2.7-km gas pipeline intrusion detection system and (b) strong periodic nuisance signal from a railway crossing for a $2.7-\mathrm{km}$ gas pipeline intrusion detection system (the railway runs perpendicularly over the pipeline).

\subsection{Pre-processing and feature extraction}

The novel nuisance alarm suppression algorithm described herein consists of event detection, signal feature extraction from the time domain representation of the signals, and a simple decision tree classifier. Event detection is based on the previously mentioned LCs algorithm. Figure 13 shows the pre-processing and feature extraction stages of the nuisance suppression algorithm.

A number of extracted features have been identified as shown in Fig. 13. Three of these features were used for nuisance alarm suppression. These features are described below:

1. Continuity of the signal: this is a measure of how continuous the signal is over its duration. It is determined by using the maximum amplitude versus segment information, $V_{m}(k)$ where $k=1,2, \ldots, K$, and $K$ is the total number of segments within the detected event (see the pre-processing stage in Fig. 13). The continuity, $C$, can be given by

$$
C=\frac{G}{K}
$$

where $G$ is the number of segments in the vector $V_{m}(k)$ with the value more than thresh $h_{2}$ and the parameter thresh $h_{2}$ is normally set above the system noise of the time domain signal as in the case of LCs. The maximum possible continuity is unity.

2. Maximum amplitude strength (MAS) (count \%): to evaluate the MAS feature, first the amplitude strength of each segment (ASES) needs to be measured. The amplitude strength relates to how much of a signal is above a given amplitude threshold thresh $h_{1}$ and is defined by (4). The parameter thresh $h_{1}$ is normally application dependent. After evaluating the amplitude strength using (4), the MAS feature is calculated as the maximum value calculated by (4) over the whole duration of the detected event (see Fig. 13). It is effectively a measure of what percentage of a signal is above a given threshold value and is given as a percentage value. This feature is important for distinguishing digging events from traffic nuisances that have similar continuity values. The intrusion signals will typically have higher maximum amplitude strengths:

$$
A S E S=\frac{S}{S_{\text {total }}} * 100
$$

where $S$ is the number of samples in the specified segment ( $>$ thresh $h_{1}$ ), and $S_{\text {total }}$ is the total number of samples in the specified segment.

3. Maximum deviation (MD, $\sigma_{m}$ ): the first step towards the evaluation of the $\mathrm{MD}$ is by the evaluation of the maximum amplitude in each segment, $V_{m}(k)$, as is the case for the continuity feature (see Fig. 13). The MD is then calculated by subtracting the mean of the vector $V_{m}(k)$ from the maximum value in the vector $V_{m}(k)$ :

$$
\sigma_{m}=\max \left(V_{m}(k)\right)-\operatorname{mean}\left(V_{m}(k)\right)
$$

where $V_{m}(k)$ is a vector containing the maximum amplitude in each segment and $k=1,2, \ldots, K$. This feature is important for discriminating between 
digging intrusion events and adjacent nuisance events of comparably long continuities, even if they have roughly similar amplitudes. In this situation, the digging events will have a higher maximum deviation owing to their higher variation in segment maxima. This can be seen by comparing the two signals represented by Figs. 11(b) (intrusion) and 12(b) (adjacent nuisance) where there are more periods of inactivity in the digging signal (lower in the amplitude) when compared with the continuous nuisance signal. This translates into a higher maximum deviation for the digging signal.

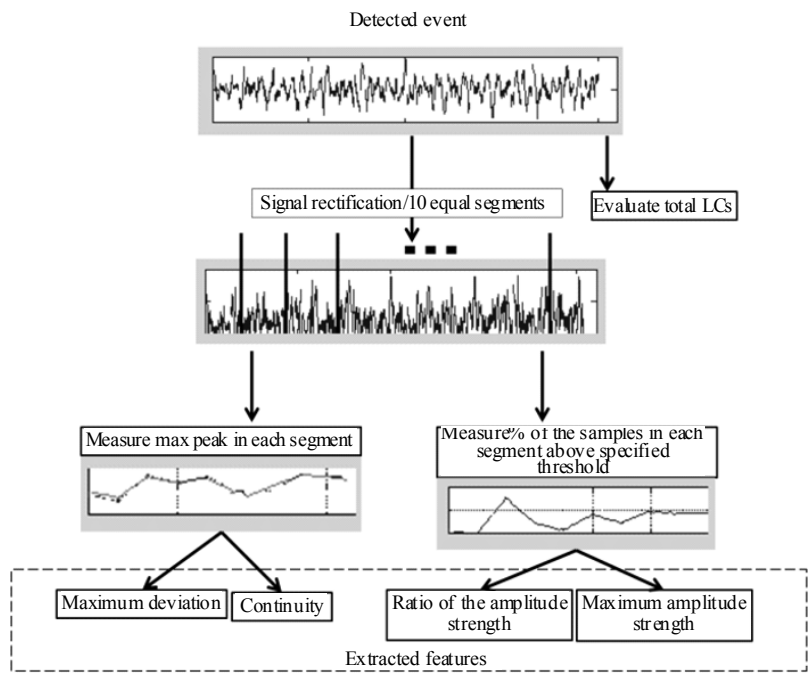

Fig. 13 New alarming system feature extraction method.

\subsection{Classification using simple decision tree}

By using the features described in the previous section in the right combination, it is possible to suppress a large number of nuisance alarms in buried intrusion detection systems. This can be done by implementing a decision tree. Decision trees represent a series of IF...THEN type rules which are linked together and can be used to classify or predict events based upon the values of a select number of features. For this work, we use a simple decision tree to discriminate between intrusion and nuisance events. Neural networks can also be used with these features to discriminate between true alarms and nuisance events. Intrusion events will generate alarms while nuisance events will be ignored.

Real captured intrusion and nuisance event signals from two sites are used to test the proposed algorithm. Table 2 shows the values of the features for the intrusion and nuisance events in Figs. 11 and 12, respectively. The real intrusion and nuisance data are used to derive appropriate threshold values, thresh_1, thresh_2, thresh_3, and thresh_4 for the decision tree as shown in Fig. 14. In this example, thresh $1=[0,0.8)$ and $[0.8,1]$, thresh $2=[0,10)$ and $[10,100]$, thresh $3=[0,15)$ and $[15,100]$ and thresh $4=[0,0.9)$ and $[0.9,5]$. Using these values the algorithm classifies digging events accurately (Figs. 11(a) and 11(b)) while traffic nuisances [such as those in Figs. 12(a) and 12(b)] are rejected.

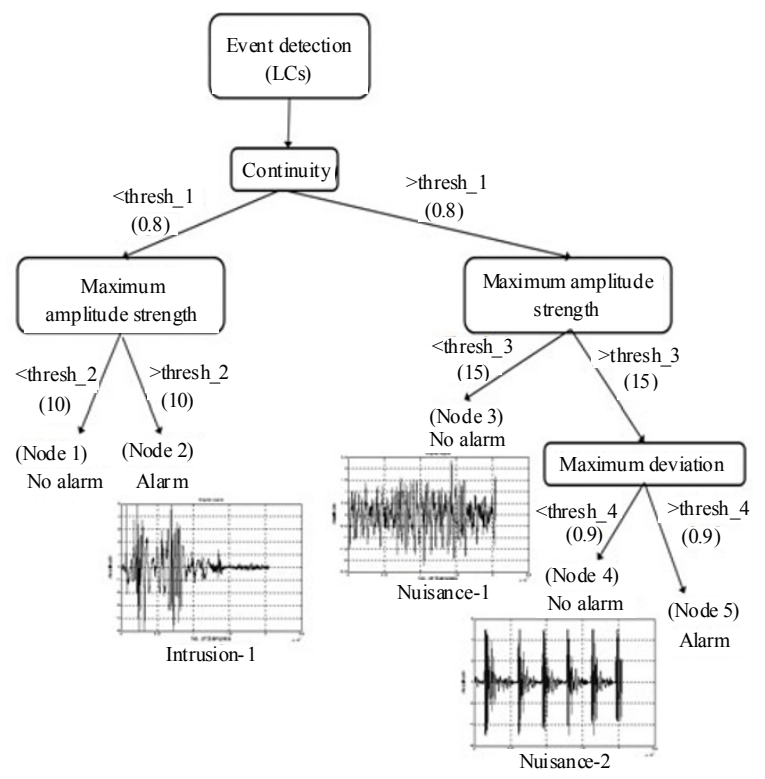

Fig. 14 Practical example of the decision tree.

Table 2 Feature values of intrusion and nuisance events.

\begin{tabular}{l|c|c|c|c}
\hline \multicolumn{5}{c|}{ Features Values } \\
\hline \multicolumn{1}{c|}{ Event Type } & $\begin{array}{c}\text { Continuity } \\
(C)\end{array}$ & MAS & $\begin{array}{c}\text { MD } \\
\left(\sigma_{m}\right)\end{array}$ & $\begin{array}{c}\text { Node } \\
\text { decision }\end{array}$ \\
\hline $\begin{array}{l}\text { Intrusion [hand and assisted } \\
\text { digging, Fig. 11(b)] }\end{array}$ & 0.4 & 34.86 & 3.595 & Node-2 \\
\hline $\begin{array}{l}\text { Vehicular traffic [adjacent } \\
\text { traffic, Fig. 12(a)] }\end{array}$ & 1 & 1.86 & 0.76 & Node-3 \\
$\begin{array}{l}\text { Road intersection and train } \\
\text { crossings [Fig. 12(b)] }\end{array}$ & 1 & 18.99 & 0.4712 & Node-4 \\
\hline
\end{tabular}




\section{Conclusions}

The performance criteria of real-time fiber optic perimeter intrusion detection systems have been discussed. Performance results have shown that the quality of sensor installation and fence construction needs to be controlled to reduce excessive nuisance signals. More importantly, an effective robust event classification system also needs to be implemented to minimize nuisance alarms while maintaining a high probability of detection to intrusion events.

A number of signal processing techniques for intrusion event detection and classification, and nuisance alarm suppression in a fiber-optic intrusion detection system have been proposed and presented. In fence-based systems, intrusion and non-intrusion events such as fence climbing, fence cutting, stick-dragging, and stone-throwing have been successfully classified in real time and demonstrated on a standard chain link fence using robust level crossings based features and artificial neural networks. Additionally, the use of a level crossings based algorithm with a dynamic threshold for suppressing torrential rain-induced nuisance alarms in fence-based fiber-optic perimeter intrusion detection systems has demonstrated its effectiveness against torrential rainfall rates in excess of $100 \mathrm{~mm} / \mathrm{hr}$. The simultaneous detection of intrusion events during rainfall periods has also been demonstrated.

In buried fiber-optic pipeline intrusion detection systems, a level crossings based detection and novel classification algorithm have also been implemented. The use of a decision tree classification algorithm has demonstrated the effective classification of both traffic induced nuisance events and digging and excavation intrusion events.

Future work is focusing on increasing the library of signal features to achieve the classification of more intrusion and nuisance events in a wider range of operating environments.
Open Access: This article is distributed under the terms of the Creative Commons Attribution License which permits any use, distribution, and reproduction in any medium, provided the original author(s) and source are credited.

\section{References}

[1] A. D. Kersey, "A review of recent developments in fiber optic sensor technology," Optical Fiber Technology, vol. 36, no. 2, pp. 291-317, 1996.

[2] J. Katsifolis and L. McIntosh, "Apparatus and method for using a counter-propagating signal method for locating events," U.S. Patent 7,499,177, 2009.

[3] J. C. Juarez, E. W. Maier, K. N. Choi, and H. F. Taylor, "Distributed fiber-optic intrusion sensor system," Journal of Lightwave Technology, vol. 23, no. 6, pp. 2081-2087, 2005.

[4] J. C. Juarez and H. F. Taylor, "Field test of a distributed fiber-optic intrusion sensor system for long perimeters," Applied Optics, vol. 46, no. 11, pp. 1968-1971, 2007.

[5] S. Tarr and G. Leach, "The dependence of detection system performance on fence construction and detector location," in Proceedings of the 32nd Annual IEEE International Carnahan Conference on Security Technology, pp. 196-200, 1998.

[6] L. H. Jiang, X. M. Liu, and F. Zhang, "Multi-target recognition used in airpoty fiber fence warning system," in Proceedings of the Ninth International Conference on Machine Learning and Cybernetics, Qingdao, Jul. 11-14, pp. 1126-1129, 2010.

[7] J. D. Vries, "A low cost fence impact classification system with neural networks," in Proceedings of 7th AFRICON Conference in Africa, Sept. 15-17, vol. 1, pp. 131-136, 2004.

[8] A. Yousefi, A. A. Dibazar, and T. Berger, "Intelligent fence intrusion detection system: detection of intentional fence breaching and recognition of fence climbing," in IEEE International Conference on Technologies for Homeland Security, Boston, May 12-13, pp. 620-625, 2008.

[9] H. Min, C. Lee, J. Lee, and C. H. Park, "Abnormal signal detection in gas pipes using neural networks," in Proceeding of 33rd Annual Conference of the IEEE Industrial Electronics, Taiwan, Nov. 5-8, pp. 2503-2508, 2007. 
[10] S. Mahmoud and J. Katsifolis, "Robust event classification for a fiber optic perimeter intrusion detection system using level crossing features and artificial neural networks," in Proc. SPIE, vol. 7677, pp. $767708,2010$.

[11] L. R. Rabiner and R. W. Schafer, Digital Processing of Speech Signals. London: Pearson Education, 1978.

[12] J. C. Junqua, B. Mak, and B. Reaves, "A robust algorithm for word boundary detection in the presence of noise," IEEE Transactions on Speech and Audio Processing, vol. 2, no. 3, pp. 407-412,
1994.

[13] S. Mahmoud and J. Katsifolis, "Elimination of rain-induced nuisance alarms in distributed fiber-optic perimeter intrusion detection systems," in Proc. SPIE, vol. 7316, pp. 731604-1-731604-11, 2009.

[14] A. Freeman and M. Skapura, Neural Networkss: Algorithms, Applications, and Programming Techniques. Massachusetts: Addison-Wesley, 1991.

[15] A. K. Jain, J. Mao, and K. M. Mohiuddin, “Artificial neural networks: a tutorial," Computer, vol. 29, no. 3, pp. 31-44, 1996. 